\title{
Identification of microscopic uranium particles using fission tracks in nuclear solid-state detectors
}

\author{
Kristína Sihelská,* Klára Řezanková, Petr Homola, František \\ Sus and Jan Lorinčík \\ Research Centre Rez

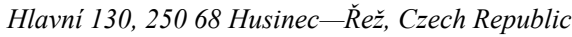 \\ *kristina.sihelska@cvrez.cz
}

Published 10 July 2018

\begin{abstract}
Improved environmental-particle analysis for safeguards is a continuing research goal. In this paper, we propose a modified method of fission-track analysis, employing the focused ion-beam (FIB) technique and using a single-crystal silicon substrate to carry dust particles. The use of a silicon substrate may eliminate the need to relocate identified particles of interest from the particle carrier to another substrate for mass-spectrometric analysis. FIB-SEM and SIMS were used to evaluate the efficiency of the proposed FTA method.
\end{abstract}

Keywords: Fission track; uranium; safeguards.

\section{Introduction}

In the 1950s, the first systematic studies of fission tracks, observed in lithium fluoride contaminated with uranium, ${ }^{1}$ were made, and transmission-electron microscopy (TEM) images in mica were published. ${ }^{2}$ In 1963, Price and Walker developed a new method for dating geological materials based on the natural decay of isotope ${ }^{238} \mathrm{U}$ by spontaneous fission; ${ }^{3}$ compared with radiography and other radiometric techniques, this method was faster, more sensitive, and able to detect lower concentrations of uranium $\left(\sim 10^{-10}\right) \cdot{ }^{4}$ In recent years, the theoretical and experimental part of the methods using tracks has been developed in fields such as geochronology, long-term monitoring of natural background radiation, the measurement of particle concentration (alpha, protons, heavy particles, cosmic rays), dosimetry, and nuclear physics.

Recently, the fission-track method has been used to detect dust particles containing fissile material in control swipes of environmental samples from nuclear facilities. ${ }^{5}$ Every process in nuclear-material handling emits tiny amounts of fissile material into the surrounding environment. Particles may be deposited in areas around and on surfaces of nuclear facilities, whether transported out and deposited on soil, vegetation, or water systems.

This is an Open Access article published by World Scientific Publishing Company. It is distributed under the terms of the Creative Commons Attribution 4.0 (CC-BY) License. Further distribution of this work is permitted, provided the original work is properly cited. 


\section{K. Sihelská et al.}

Every hot particle carries information about its chemical, radiological, and even metallurgical history, providing invaluable information about the origin, production process, and potential use of the source material..$^{5}$ This information is contained in the isotopic composition of individual particles and can be obtained using massspectrometric techniques such as secondary-ion mass spectrometry (SIMS), thermalionization mass spectrometry (TIMS), or inductively-coupled-plasma mass spectrometry (ICP-MS).

An important preliminary step before conducting isotopic analysis of individual particles is to determine their location and identification in a sample covered with a large quantity of nonspecific environmental dust. While particle screening can be performed using scanning electron microscopy combined with energy-dispersive X-ray spectroscopy (SEM-EDX), ${ }^{6}$ SIMS is the only method that both localizes the particles and provides their isotopic composition. ${ }^{7}$ There are several factors limiting the performance of those two particle-screening methods. First, SIMS is destructive-it erodes material in particlesearch imaging mode, reducing the quantity of particle material available and potentially lowering analytical precision in the case of very small particles of interest. Moreover, the interior of the SIMS instrument may become contaminated by unwanted sputter deposits of radioactive material. The efficacy obtained using a SEM-EDX in automated particlesearch mode is subject to the settings and stability of the instrument, potentially leading to overlooked particles of interest.

Fission-track analysis (FTA) is a third method of locating particles containing nuclear material, ${ }^{8}$ offering extraordinary sensitivity, objectivity, and conclusiveness in the detection and identification of tested nuclear material. Typically for FTA, particles are extracted from cotton swipes, embedded in a film consisting of a solution of ethanol and collodion, and attached to a nuclear solid-state detector. The whole assembly is then exposed for several minutes to thermal neutron flux in a research nuclear reactor.

The detector is separated and etched using a $\mathrm{NaOH}$ solution to enlarge latent tracks from the interaction of high-energy fission products with the detector. Observed through an optical microscope, the positions of star-shaped latent-track clusters reveal the positions of fissile nuclei. Selected particles are removed from the film and typically placed onto filaments for TIMS. ${ }^{9}$ FTA has been adopted by several laboratories and research groups (see Refs. 10-13) and, besides TIMS, has also been coupled with SIMS (see Ref. 14) or ICP-MS (see Ref. 15).

Demand continues for improved nuclear forensics in terms of speed, sensitivity, and reliability. In this contribution, we describe a version of FTA characterized by employing the focused ion-beam (FIB) technique and using a single-crystal silicon substrate as a carrier of the dust particles. We combine the FTA with SIMS to evaluate the proposed FTA procedure and estimate the isotopic composition of the particles. 


\section{Fission Track Analysis Procedure}

\subsection{Sample-assembly preparation}

Uranium-oxide particles $\left(\mathrm{U}_{3} \mathrm{O}_{8}\right)$ from certified reference material SRM U030 (National Institute of Standards and Technology, Washington DC) with 3\% ${ }^{235} \mathrm{U}$ enrichment, a particle size of 1-5 $\mu \mathrm{m}$, and a dust mixture were used. The particles were added to a solution of collodion and ethanol of a 1:100 volume ratio. A high-purity silicon wafer was cut to $10 \mathrm{~mm} x 15 \mathrm{~mm}$, and $5 \mu \mathrm{l}$ of uranium suspension was pipetted onto the silicon substrate and dried in a laminar box at room temperature to form a particle layer. A Lexan polycarbonate detector (Goodfellow Cambridge Ltd.) with a thickness of $375 \mu \mathrm{m}$ was cut by laser to the same size as the silicon substrate.

The laser was used to drill three 200- $\mu$ m-diameter holes to define the coordinate system of the Lexan detector. A small area around the holes was covered with a thin layer of gold in a sputter coater to avoid charging effects during FIB-SEM processing and later removed before insertion into the neutron field. The detector was attached to the $\mathrm{Si}$ substrate covered with particles and fixed with plexiglass clamps and plastic screws to form a sample assembly (see Fig. 1).

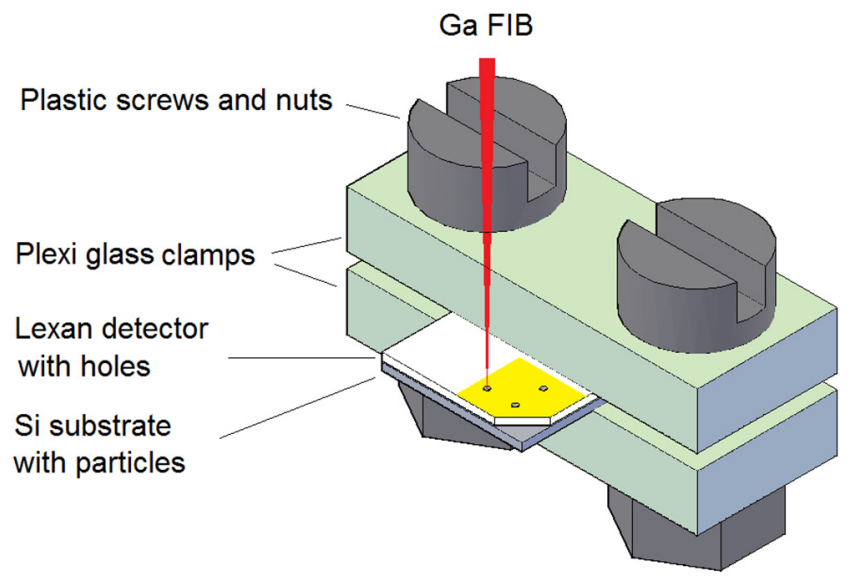

Fig. 1. The sample assembly consisting of a Si substrate with deposited dust particles, the attached Lexan detector, two plexiglass clamps, and plastic screws. The Ga-ion beam indicates the procedure of forming fiducial marks in Si through the holes in the detector. The open triangular area on the Si served for making test marks.

The sample assembly was inserted into the FIB-SEM instrument (a Lyra3 from Tescan), and fiducial marks (see Fig. 2, Left) were milled using a $\mathrm{Ga}^{+}$beam at $30 \mathrm{keV}$, $1 \mathrm{nA}$, and $\sim 100 \mathrm{~nm}$ spot size. The Ga beam was directed through the center of the holes in the Lexan detector (see Fig. 1). The exact overlap of the centers with the centers of the three marks in the Si substrate formed the basis for synchronizing their coordinate systems (see Fig. 2). 


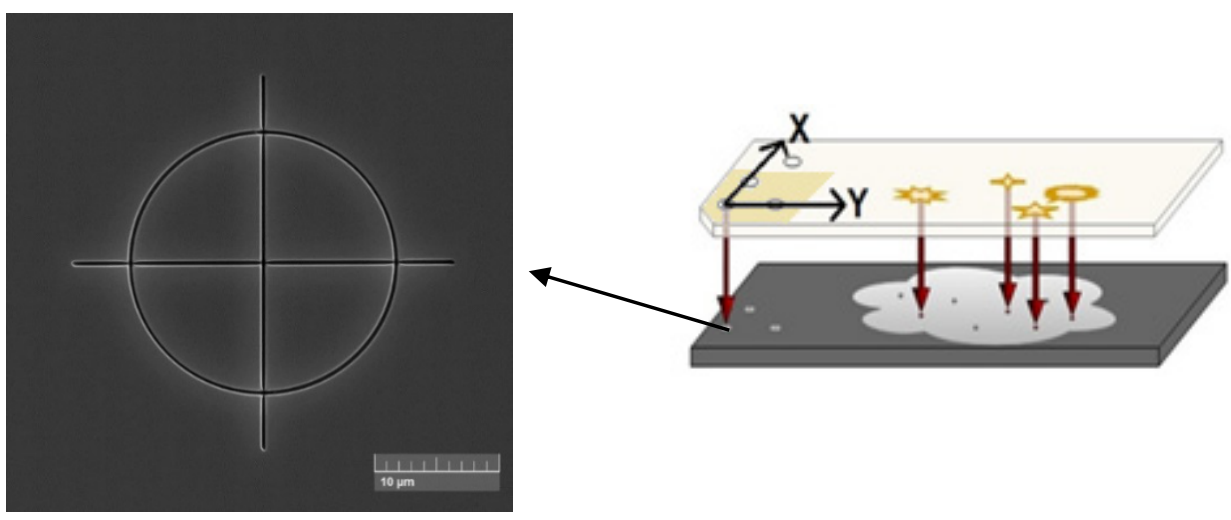

Fig. 2. Left: SEM micrograph of a fiducial mark made in Si substrate with the Ga FIB. Right: Drawing of the synchronization of the coordinate systems of the detector and the Si substrate. Objects in yellow represent fission-track clusters. The dots below are corresponding uranium-oxide particles.

\subsection{Thermal neutron irradiation}

The sample assembly was placed in an irradiation capsule made of polyethylene, inserted into research nuclear reactor LVR-15 at the Research Centre Rez, Czech Republic, and exposed to a thermal neutron-flux rate of $3.2 \times 10^{13} \mathrm{~cm}^{-2} \mathrm{~s}^{-1}$ for two minutes. Samples were transported to the reactor core via pneumatic tube.

\subsection{Processing of the detectors and identification of uranium particles}

After irradiation, the clamps were removed from the sample assemblies, and the detectors were separated from the silicon substrates. The detectors were etched in $6.5 \mathrm{M} \mathrm{NaOH}$ at $65^{\circ} \mathrm{C}$ for $30 \mathrm{~min}$, rinsed with purified water, and dried in the laboratory drier for $10 \mathrm{~min}$. In this way, the tracks were enlarged to sizes easily observable with an optical microscope or even the unaided eye in the case of large track clusters.

Optical images with a $4 x$ objective were taken in transmitted light and bright-field mode and digitally combined into a macro-photograph of the whole detector area (see Fig. 3). The macro-photograph was then processed in image-analysis software. The origin of the coordinate system was placed in the central hole, and the $x$ and $y$ coordinates of all track clusters were recorded manually by pointing and clicking on the cluster centers. These coordinates were directly transferable to the particle coordinates on the substrate.

\subsection{Isotopic ratio measurement}

After obtaining the coordinates of the particles of interest from the optical composite photograph, the Si substrates with particles were inserted into the SIMS instrument (IMS 7f, CAMECA) without relocating the particles onto another substrate. The SIMS sample stage was moved so that the central mark on the substrate was placed on the optical axis 
of the secondary-ion optics. The fiducial marks from Fig. 2 were large enough to be visible with the optical microscope in SIMS.
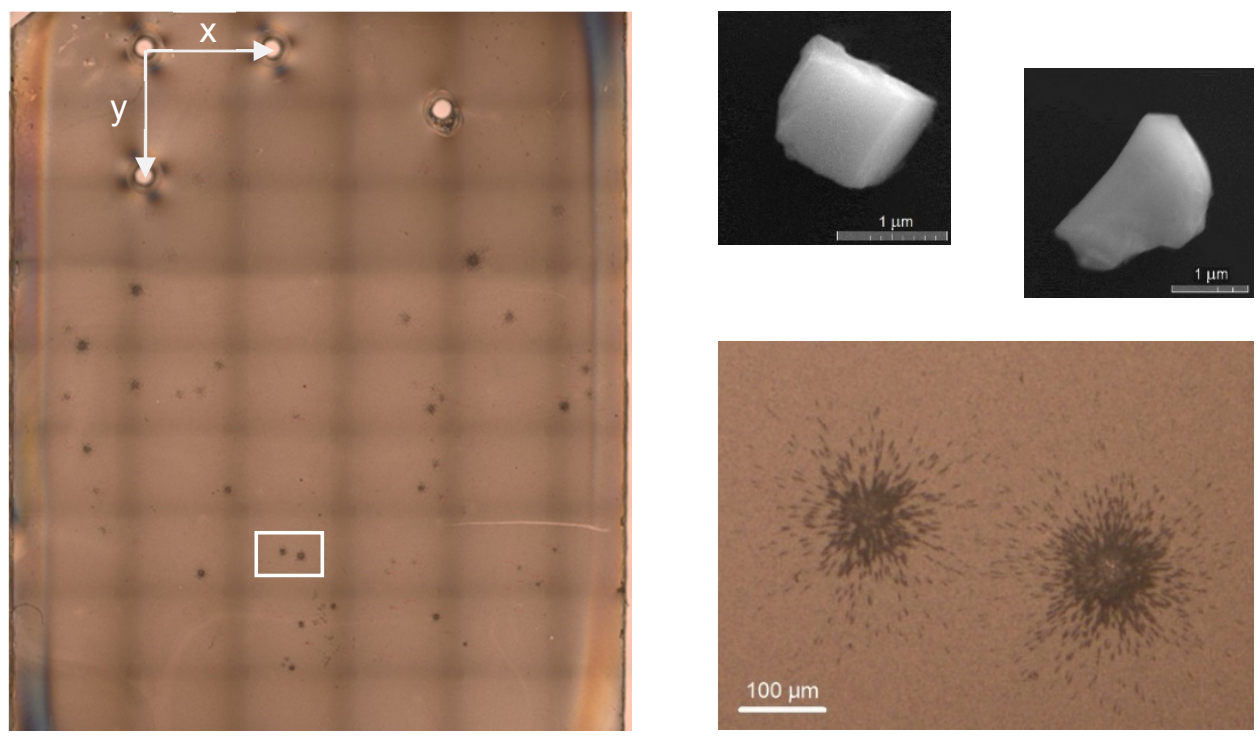

Fig. 3. Left: optical composite photograph of fission tracks made of 54 view fields $1.4 \mathrm{~mm} \times 1.6 \mathrm{~mm}$. Right: an enlarged image of two fission-track clusters (bottom) and SEM images of the corresponding uranium-oxide particles (top).

Because we expected an uncertainty on the order of tens of micrometers in the navigation to the particle position, we first took a $100 \mu \mathrm{m} x 100 \mu \mathrm{m}$ ion image of ${ }^{238} \mathrm{U}$. From that image, we determined the geometrical deviation of the particle position from the optical axis and made fine adjustments to the sample stage. Next, the spectrometer was set for depth-profiling mode. A $10 \mathrm{keV} \mathrm{O}_{2}{ }^{+}$primary ion beam was raster-scanned over an area of $25 \mu \mathrm{m} x 25 \mu \mathrm{m}$, centered on the particle. The $\mathrm{O}_{2}{ }^{+}$current was $1 \mathrm{nA}$. The instrument was set for mass resolution $\mathrm{M} / \Delta \mathrm{M} \sim 400$. Positively charged secondary ions ${ }^{234} \mathrm{U}^{+},{ }^{235} \mathrm{U}^{+},{ }^{236} \mathrm{U}^{+},{ }^{238} \mathrm{U}^{+}$, and ${ }^{238} \mathrm{UH}^{+}$were recorded. ${ }^{238} \mathrm{UH}^{+}$was recorded to correct the ${ }^{236} \mathrm{U} /{ }^{238} \mathrm{U}$ ratios due to the mass interference of ${ }^{235} \mathrm{UH}^{+}$and ${ }^{236} \mathrm{U}^{+}$.

It should be noted, that the product of the reaction cross-section and the thermal neutron flux is very small for ${ }^{235} \mathrm{U}$ nuclei $\left(\sim 10^{-6} \mathrm{~s}^{-1}\right)$, therefore the influence of the ${ }^{235} \mathrm{U}$ reduction after the irradiation on the accuracy of the isotopic ratios can be neglected.

\section{Results and Discussion}

The procedure for sample-assembly preparation was repeated several times with a high level of reproducibility and could be further optimized, e.g., by using another plastic detector (a thicker detector is recommended in Ref. 15).

The irradiation conditions should be optimized for reliable identification and good observability of fission tracks, which requires taking into account anticipated share of 
${ }^{235} \mathrm{U}$ in the microscopic volumes of particles. In this work, the irradiation time and neutron flux were optimized for low enriched uranium-oxide particles $(0.71-20 \%)$. Depleted $(<0.71 \%)$ or highly enriched uranium $(\geq 20 \%)$ will require longer or shorter irradiation times on LVR-15, respectively.

The motorized stage of the optical microscope allowed for the fast creation, within minutes, of a large composite photograph comprising tens of view fields. The coordinate recording was made manually by pointing and clicking, which is reasonably fast if the number of particles is not too high $(<100)$. For a larger number of tracks, an automated search for the center of the fission-track clusters would be necessary.

The FIB-SEM system was used to estimate the performance parameters of the modified FTA. The composite photograph was uploaded to the FIB-SEM-EDX system and synchronized with the SEM stage. This allowed movement to the particle location by pointing and clicking on the fission-track-cluster center in the uploaded image. Alternatively, the coordinates of the particles may be typed into the stage-control panel.

Two parameters are used for evaluating the performance of the procedure: the success ratio and location accuracy (see Ref. 15). The success ratio is defined as the ratio of the identified particles of interest and the total number of identified fission-track clusters. Location accuracy can be understood as the geometrical error between the true position of the particle on the substrate and the location predicted from the optical image of the fission tracks. The success ratio in the proposed procedure was $100 \%, \sim 80 \%, \sim 50 \%$ in $40 \%, 30 \%, 30 \%$ experiments, respectively. The probable cause for the low success ratio in the $30 \%$ cases was loss of particles or their substantial $(>100 \mu \mathrm{m})$ relocation during manipulation with the sample. The location accuracy was between $20 \mu \mathrm{m}$ and $100 \mu \mathrm{m}$ for both SEM and SIMS. This accuracy was insufficient for the isotopic ratio measurement in SIMS; therefore a medium-sized scan in imaging or retrospective analysis mode was performed before fine stage positioning (centering of the particle on the optical axis).

The isotopic ratios were determined from the flat parts of the SIMS depth profiles with the following values averaged over nine particles:

- ${ }^{234} \mathrm{U} /{ }^{238} \mathrm{U}=2.0 \times 10^{-4}$

- $\quad{ }^{235} \mathrm{U} /{ }^{238} \mathrm{U}=3.2 \times 10^{-2}$

- ${ }^{236} \mathrm{U} /{ }^{238} \mathrm{U}=4.4 \times 10^{-4}$

Deviation from nominal expectations was $\sim 3 \%$ for ${ }^{234} \mathrm{U} /{ }^{238} \mathrm{U}$ and ${ }^{235} \mathrm{U} /{ }^{238} \mathrm{U}$ ratios and a factor of 2 for ${ }^{236} \mathrm{U} /{ }^{238} \mathrm{U}$. The latter deviation may be due to mass interferences. As the main purpose of the SIMS measurements was to evaluate the proposed FTA procedure, no attempt was made to improve the accuracy of isotopic ratios.

\section{Summary}

This study demonstrates a modification to the FTA for single-particle identification, namely, the use of the FIB technique in sample-assembly preparation and of a singlecrystal silicon surface as a uranium-particle carrier. Using a silicon substrate can 
eliminate the need for relocating the identified particles of interest from the particle carrier to another substrate for mass-spectrometric analysis. Under this approach, submicrometer sized uranium-oxide particles may be identified and micro-probed. The largest delay encountered with this method is in the cooling and clearing of irradiated samples (several days). The other analytical steps (assembly preparation, detector processing, SIMS analysis) took about a day each.

\section{Acknowledgments}

The presented work was financially supported by the Ministry of Education, Youth and Sport, Czech Republic Project LQ1603 (Research for SUSEN). This work has been performed within the SUSEN Project established in the framework of the European Regional Development Fund (ERDF) in project CZ.1.05/2.1.00/03.0108. The sample irradiation was carried out at the CANAM infrastructure of the NPI CAS Rez supported through the Ministry of Education, Youth and Sport, Czech Republic Project No. LM2015056.

\section{References}

1. D. A. Young, Nature 182, 375 (1958).

2. E. C. H. Silk and R. S. Barnes, Philos. Mag. 4, 970 (1959).

3. G. Wagner and P. Van den Haute, Fission-Track Dating (Springer, Netherlands, 1992).

4. H. S. Virk and S. Singt, Mineral. Journal 8, 263 (1977).

5. D. Donohue, J. All. Comp. 271-273, 11 (1998).

6. F. Esaka and M. Magara, Mass Spectrom. Lett. 7, 41 (2016).

7. P. Peres, P. M. L. Hedberg, S. Walton, N. Montgomery, J. B. Cliff, F. Rabemananjaraa and M. Schuhmacher, Surf. Interface Anal. 45, 561 (2013).

8. O. Stetzer, M. Betti, J. Geel, J. V. K. Erdmann, R. Schenkel and N. Trautmann, Nuc. Instr. Meth. Phys. Res. A525, 582 (2004).

9. F. Esaka, D. Suzuki and M. Magara, Anal. Chem. 87, 3107 (2015).

10. C. G. Lee, D. Suzuki, F. Esaka, M. Magara, N. Shinohara and S. Usuda, J. Nuc. Scien. Tech. 46, 809 (2009).

11. M. H. Lee, J. H. Park and K. Song, Radiat. Meas. 46, 409 (2011).

12. T. Yang, L. Luo, X. Liu, X. Shao, C. Wang, L. Tang and K. Long, Raioanal. Nucl. Chem. 309, 461 (2016).

13. Y. Chen, Y. Shen, Z. Y. Chang, Y. G. Zhao, S. L. Guo, J. Y. Cui and Y. Liu, Radiat. Meas. 50, 43 (2013).

14. F. Esaka, C.-G. Lee, M. Magara and T. Kimura, Anal. Chim. Acta 721, 122 (2012).

15. N. Dzigal, E. Chinea-Cano, S. Walsh and A. Limbeck, Talanta 167, 583 (2017). 